NASZA DERMATOLOGIA Online OUR DERMATOLOGY Online

Source of Support: Nil

Competing Interests:

None declared

\section{PUSTULAR PSORIASIS RESPONDING TO PROBIOTICS - A NEW INSIGHT}

\author{
Metikurke Vijayashankar, Nithya Raghunath
}

MVJ Medical College and Research Hospital, Bangalore, India

Corresponding author: Dr. Nithya Raghunath

nithyareddy85@gmail.com

\begin{abstract}
Probiotics exhibit widespread effects on homeostasis and immunomodulation of both mucosal and systemic immunity. Probiotics counter weight aggressive commensals in the body and reinforce the barrier function of the epithelium while also contributing to the regulation of innate and adaptive immune responses of the host under healthy or pathogenic conditions. Probiotics could be used for prevention or treatment of chronic allergic and inflammatory diseases, such as inflammatory bowel disease (IBD) and atopic dermatitis. We describe a case of pustular psoriasis where probiotics were used for the treatment successfully.
\end{abstract}

Key words: psoriasis; pustular psoriasis; probiotics

\section{Introduction}

Probiotics exhibit widespread effects on homeostasis and immunomodulation of both mucosal and systemic immunity. Probiotics counter weight aggressive commensals in the body and reinforce the barrier function of the epithelium while also contributing to the regulation of innate and adaptive immune responses of the host under healthy or pathogenic conditions. Probiotics could be used for prevention or treatment of chronic allergic and inflammatory diseases, such as inflammatory bowel disease (IBD) and atopic dermatitis. We describe a case of pustular psoriasis where probiotics were used for the treatment successfully.

\section{Case Report}

A 47yr old female, presented with crops of pustules all over her body since 20 days. The rashes started over the leg and rapidly progressed to the whole body. The rashes were associated with pain. Patient had fever and arthritis of both knees. There was no photosensitivity. Patient was a known case of psoriasis since 15 years. She was on treatment with different medications both topical and systemic. She was given Methotrexate 2yrs back for 6 months and since then she was on irregular treatment. The patient was anxious, looked very ill and was febrile. General physical examination showed bilateral pedal edema. On cutaneous examination there was diffuse erythema with areas of pustules over extensor aspect of upper and lower limbs, abdomen and chest (Fig. 1,2). Multiple scaly plaques were present over the scalp. Axilla and groins had no lesions. Nails showed pitting with subungual hyperkeratosis. Palms and soles were normal. Systemic examination was within normal limits.

Her random blood sugar was $220 \mathrm{mg} / \mathrm{dl}$, triglycerides were $344 \mathrm{mg} / \mathrm{dl}$, C-Reactive Protein was positive, total protein was $5.6 \mathrm{~g} / \mathrm{dl}$ and albumin was $3 \mathrm{~g} / \mathrm{dl}$. Ultrasonography of the abdomen did not show any significant abnormality.

On the basis of history, clinical examination a diagnosis of pustular psoriasis was made. The patient was admitted and put on steroids, dapsone and as she was already on methotrexate, it was continued with supplementation of analgesics and antipyretics. But even after two weeks she did not respond and her lesions kept increasing and her blood sugar increased. She started developing signs of steroid toxicity. We were forced to withdraw steroids. As she could not afford biologics we had to look for alternative medicines.

With anecdotal reference [4] of probiotics helping palmoplantar pustular psoriasis and their usefulness in atopic dermatitis, we thought of trying the regimen after obtaining consent from the patient. The patient was put on Lactobacillus, one sachet thrice daily with biotin 10mg once daily. All the other drugs were stopped forthwith. In fifteen days the fever subsided, lesions started involuting and no new lesions appeared (Fig. 3, 4). The patient's general condition also showed improvement. Blood sugar level dropped. She was continued with the same treatment and after six months follow up she is free of lesions and she is being followed up for possible recurrence. Her plaque psoriasis is also under control with only salicylic acid and coal tar. 

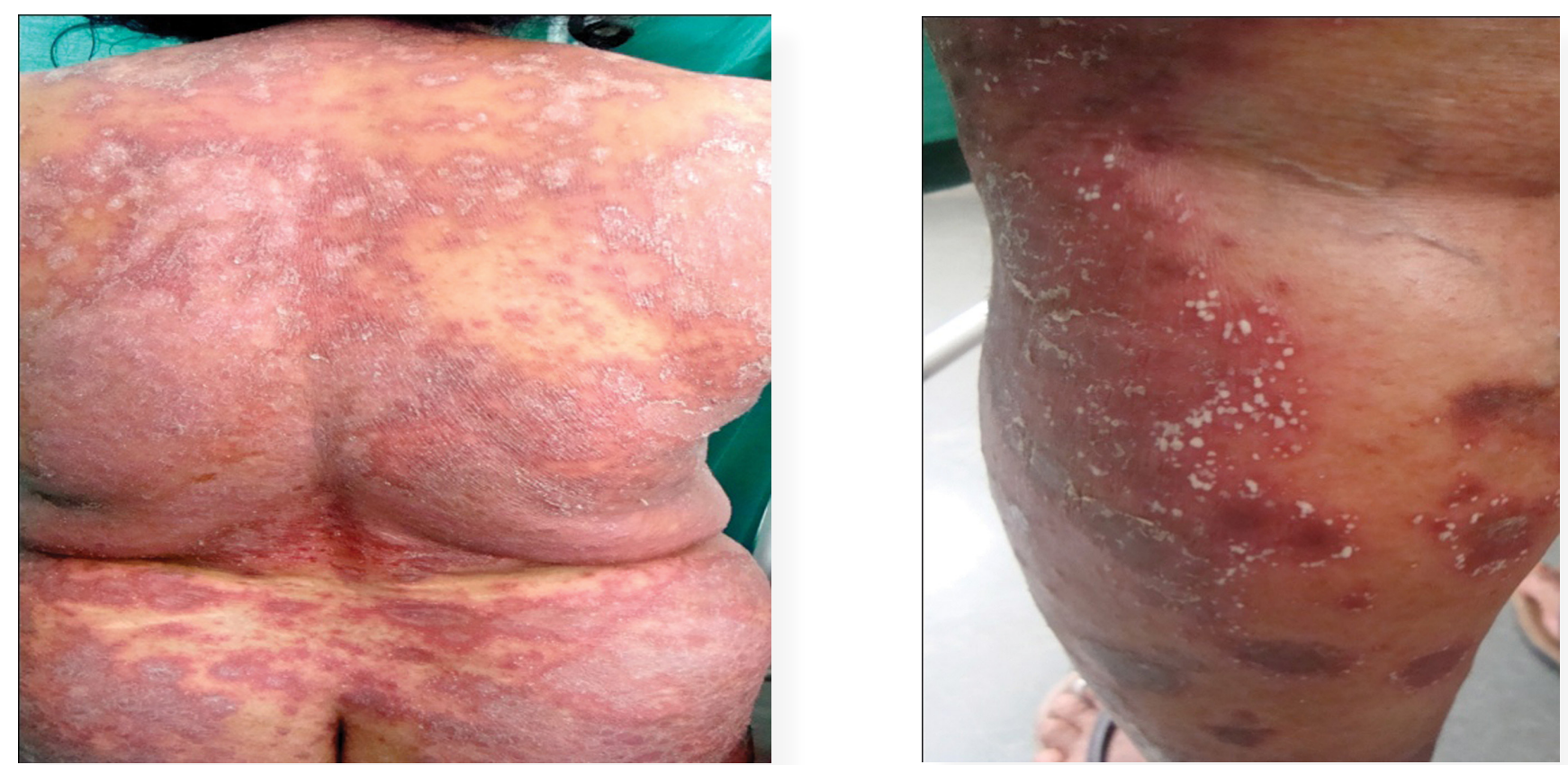

Figure 1 and 2. Pre-treatment: Pustular lesions over the back and leg prior to the treatment with probiotics
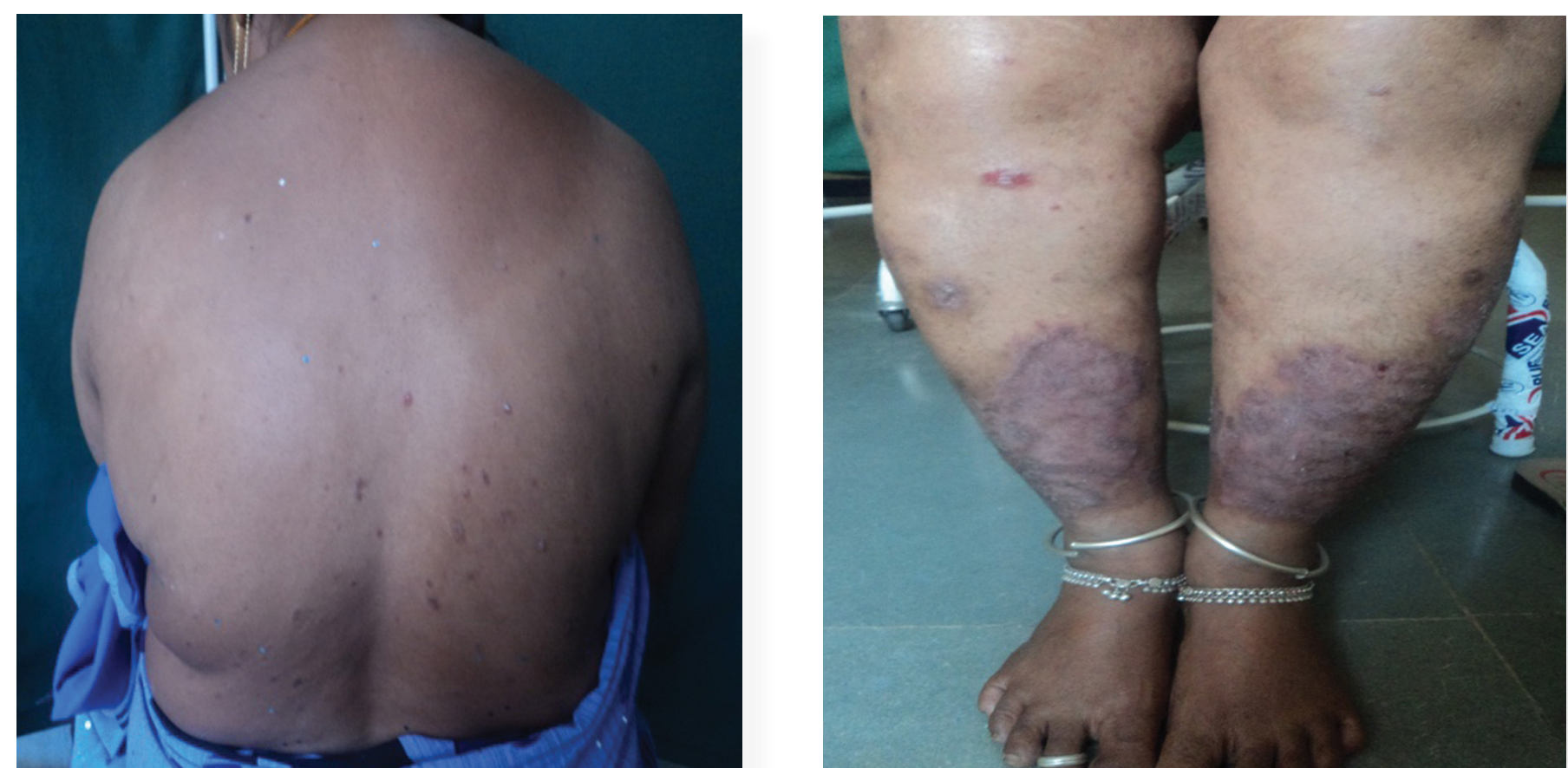

Figure 3 and 4. Post-treatment: Healed pustular lesions with few erythematous plaques over back and legs after 4 weeks of treatment

\section{Discussion}

Administration of probiotic Lactobacillus strains (a mixture of Lactobacillus rhamnosus 19070-2 and Lactobacillus reuteri DSM 12246) to children with atopic dermatitis has been shown to result in a moderate improvement in the clinical severity [5].

It is being reported that most of the probiotics containing lacticacid producing bacteria (LAB) strains are nonpathogenic and nontoxigenic microorganisms. Over 70 clinical studies have been conducted over food containing microbial ingredients to investigate potential probiotic-side effects.
None have shown any adverse effects [6]. The rationale of usage of such probiotics is furthered by its potential multipronged action. Though unclear the proposed mechanisms of action include modulation of immune response in the gut which can directly affect the development of inflammatory disease mechanisms in systemic sites of disease, such as the skin [7]. However, the mechanisms by which intestinal immune responses translate to systemic anti-inflammatory effects remain to be established. In our case Lactobacillus Sporogenes was used as it was the only available lactobacilli in the market along with biotin. 
However, to date, the overall clinical evidence on the benefits of probiotics in the management of systemic inflammatory diseases is inconclusive. It is likely that quantity and time of administration, as well as the interaction with specific components of the host's microflora, will heavily influence the immunomodulatory effects of probiotics. The situation is complicated by the fact that different probiotic strains have different immunomodualtory patterns. Our treatment success in this case though forlorn can act as a catalyst in further evaluation of this exciting prospect in the management of psoriasis.

\section{Acknowledgements}

Dr. Sujatha C, Prof. and Head Dept. of Dermatology, MVJ Medical College and Research Hospital.

Dr. Vasantha Kumar S, Principal, MVJ Medical College and Research Hospital.

\section{REFERENCES}

1. Boirivant M, Strober W: The mechanism of action of probiotics. Curr Opin Gastroenterol. 2007;23:679-92.

2. Sartor RB: Therapeutic manipulation of the enteric microflora in inflammatory bowel diseases: antibiotics, probiotics, and prebiotics. Gastroenterology. 2004;126:1620-33.

3. Lee J, Seto D, Bielory L: Meta-analysis of clinical trials of probiotics for prevention and treatment of pediatric atopic dermatitis. J Allergy Clin Immunol. 2008;121:116-21, e111.

4. Dr. Masaru Maebashi: www.inspire.com and http://www3.ocn. ne.jp/ hpps/english/english.html. Downloaded 3.00 pm 0n 08-0512.

5. Rosenfeldt V, Eva Benfeldt, Nielsen SD, Michaelsen KF, Jeppesen DL, Valerius NH, et al: Effect of probiotic Lactobacillus strains in children with atopic dermatitis. J Allergy Clin Immunol. 2003;111:389-95.

6. Saavedra, Jose M: Use of Probiotics in Pediatrics: Rationale, Mechanisms of Action, and Practical Aspects. Nutr Clin Pract. 2007;22:351-65.

7. Ly NP, Litonjua A, Celedon JC, Gold DR: Gut microbiota, probiotics, and vitamin D: interrelated exposures influencing allergy, asthma, and obesity? J Allergy Clin Immunol. 2011;127:1087-94.

Copyright by Metikurke Vijayashankar, et al. This is an open access article distributed under the terms of the Creative Commons Attribution License, which permits unrestricted use, distribution, and reproduction in any medium, provided the original author and source are credited. 